\title{
Interface Strength Measurements
}

\author{
VIKAS TOMAR ${ }^{1,3}$ and RITESH SACHAN ${ }^{2,4}$ \\ 1.-Purdue University, West Lafayette, IN, USA. 2.-Oak Ridge National Laboratory, Oak Ridge, \\ TN, USA. 3.—e-mail: Tomar@purdue.edu. 4.—e-mail: sachanr@ornl.gov
}

Interfaces are ubiquitous in all engineering materials in the form of grain boundaries and phase boundaries in metals and alloys, intergranular thin films in ceramics, and interphases in polymer composites (collectively referred to as interfaces). Interfaces in materials are usually at appreciably smaller scale thicknesses (nanometers in metals to micrometers in polymer composites with intergranular thin films inbetween) than the primary and secondary phases, yet a slight change in their chemistry leads to significant changes in material behavior, especially when failure in extreme environments is considered (extreme strain rates $>10^{3} \mathrm{~s}^{-1}$, homologous temperature $>0.4$, corrosive environments, high moisture, low temperature, etc.). Research into the effect of interfaces on material deformation and failure has become increasingly significant as advancements in engineering materials and structures have necessitated their operation in extreme environments. This topic consists of research papers focusing on interface strength measurements and the incorporation of such measurements for predicting the important roles that interfaces play in determining material behavior.

The research article by Naya Montáns et al. focuses on using a push-in test-based experimental methodology combined with finite element analyses to predict the shear interface strength of fiberreinforced polymers. Examples of applications are presented to determine the shear interface strength in carbon and glass fiber composites reinforced with either thermoset or thermoplastic matrices. Interface strength measurements must be accompanied with high-throughput analyses and uncertainty characterization in order to predict interface property influences on the mechanical behavior of materials. The review article by Kalidindi et al. focuses on presenting and summarizing such an approach. Interface complexion evolves during material processing. Correspondingly, a structural material's properties are significantly linked to interface evolution. A review article by Verma et al. focuses on recent work carried out in connecting interface evolution to the interface mechanics using a combination of phase field modeling, ab initio modeling, and finite element analyses.

The presented articles' approach is different from a "material science"-based approach which fundamentally focuses on interface-based deformation mechanisms, interface thermodynamics, and interface chemistry. Overall, the articles in this "interface strength measurements" topic focus on different aspects of "mechanics of materials" that emphasize links between mechanism-based interface mechanical properties and the mechanical behavior of materials.

The following papers being published under the topic of interface strength measurements provide excellent details and research on the subject. To download any of the papers, follow the url http:// link.springer.com/journal/11837/69/1/page/1 to the table of contents page for the January 2017 issue (vol. 69, no. 1).

- "Interface Characterization in Fiber-Reinforced Polymer-Matrix Composites" by F. Naya Montáns, J.M. Molina-Aldareguía, C.S. Lopes, C. González, and J. LLorca.

- "Mechanical Characterization of Mesoscale Interfaces Using Indentation Techniques" by Surya R. Kalidindi, Soumya Mohan, and Alicia Rossi.

- "Relating Interface Evolution to Interface Mechanics Based on Interface Properties: A Review" by Devendra Verma, Sudipta Biswas, Chandra Prakash, and Vikas Tomar. 